\title{
MEAN VALUE THEOREMS FOR AUTOMORPHIC $L$-FUNCTIONS
}

\author{
O. M. FOMENKO
}

Dedicated to the 100th anniversary of D. K. Faddeev's birth

\begin{abstract}
Let $f$ be a holomorphic Hecke eigencuspform of even weight $k \geq 12$ for $\operatorname{SL}(2, \mathbb{Z})$ and let $L\left(s, \operatorname{sym}^{2} f\right)$ be the symmetric square $L$-function of $f$. Let $C(x)$ be the summatory function of the coefficients of $L\left(s, \operatorname{sym}^{2} f\right)$. The true order is found for

$$
\int_{0}^{x} C(y)^{2} d y
$$
\end{abstract}

\section{$\S 1$. Introduction. The Results}

Consider the space $S_{k}(\Gamma)$ of holomorphic cuspforms

$$
f(z)=\sum_{n=1}^{\infty} a_{f}(n) e(n z)
$$

of even weight $k \geq 12$ for the group $\Gamma:=\mathrm{SL}(2, \mathbb{Z})$, where $\mathbb{Z}$ is the ring of rational integers, $z=x+i y, y>0$, and $e(\xi):=e^{2 \pi i \xi}$. Let $S_{k}(\Gamma)^{+}$be the set of all Hecke eigencuspforms in this space with $a_{f}(1)=1$. It is known that $S_{k}(\Gamma)=\varnothing$ if $k$ is odd, or if $k$ is even and $k<12$.

From now on, we assume that $f \in S_{k}(\Gamma)^{+}$. Set

$$
\lambda_{f}(n)=a_{f}(n) / n^{\frac{k-1}{2}} ;
$$

$\lambda_{f}(n)$ is an eigenvalue of the Hecke operator $T(n), n=1,2,3, \ldots$. By the classical result by Deligne, for a prime $p$ we have

$$
\lambda_{f}(p)=\alpha_{p}+\bar{\alpha}_{p}, \quad \alpha_{p} \bar{\alpha}_{p}=1 .
$$

We consider the following automorphic $L$-functions, initially defined in the half-plane $\sigma>1$ (as always, $s=\sigma+i t)$ :

$$
L(s, f):=\prod_{p}\left(1-\alpha_{p} p^{-s}\right)^{-1}\left(1-\bar{\alpha}_{p} p^{-s}\right)^{-1}=\sum_{n=1}^{\infty} \lambda_{f}(n) n^{-s}
$$

(the Hecke $L$-function of $f$ );

$$
\begin{aligned}
L\left(s, \operatorname{sym}^{2} f\right) & :=\prod_{p}\left(1-\alpha_{p}^{2} p^{-s}\right)^{-1}\left(1-p^{-s}\right)^{-1}\left(1-\bar{\alpha}_{p}^{2} p^{-s}\right)^{-1} \\
& =\zeta(2 s) \sum_{n=1}^{\infty} \lambda_{f}\left(n^{2}\right) n^{-s}=: \sum_{n=1}^{\infty} c_{n} n^{-s}
\end{aligned}
$$

2000 Mathematics Subject Classification. Primary 11M41.

Key words and phrases. Symmetric square $L$-function, summatory function, Euler product, Voronoi formula, mean value. 
(Shimura's symmetric square $L$-function of $f$, see [1]); here $\zeta(s)$ is the Riemann zeta function;

$$
\begin{aligned}
L(s, f \times f) & :=\prod_{p}\left(1-\alpha_{p}^{2} p^{-s}\right)^{-1}\left(1-p^{-s}\right)^{-1}\left(1-p^{-s}\right)^{-1}\left(1-\bar{\alpha}_{p}^{2} p^{-s}\right)^{-1} \\
& =\zeta(2 s) \sum_{n=1}^{\infty} \lambda_{f}(n)^{2} n^{-s}=: \sum_{n=1}^{\infty} d_{n} n^{-s}
\end{aligned}
$$

(the $L$-function of the Rankin-Selberg convolution of $f$ with itself [2, 3]).

There are more complicated Euler products, for example,

$$
L\left(s, \operatorname{sym}^{m} f\right):=\prod_{p} \prod_{j=0}^{m}\left(1-\alpha_{p}^{m-j} \bar{\alpha}_{p}^{j} p^{-s}\right)^{-1} \quad(\sigma>1),
$$

where $m=3,4,5, \ldots$ (see [4]), but at the moment they are less investigated.

The $L$-functions introduced above admit analytic continuation to the entire complex plane, $L(s, f)$ and $L\left(s, \operatorname{sym}^{2} f\right)$ are entire functions, and $L(s, f \times f)$ has a simple pole with residue $R$ at $s=1$ as its only singularity. Each of these $L$-functions possesses a functional equation connecting $s$ and $1-s$.

In what follows we use the functional equation for $L\left(s, \operatorname{sym}^{2} f\right)$ :

$$
\begin{aligned}
\pi^{-\frac{3}{2} s} & \Gamma\left(\frac{s}{2}+\frac{1}{2}\right) \Gamma\left(\frac{s}{2}+\frac{k-1}{2}\right) \Gamma\left(\frac{s}{2}+\frac{k}{2}\right) L\left(s, \operatorname{sym}^{2} f\right) \\
& =\pi^{-\frac{3}{2}(1-s)} \Gamma\left(\frac{1-s}{2}+\frac{1}{2}\right) \Gamma\left(\frac{1-s}{2}+\frac{k-1}{2}\right) \Gamma\left(\frac{1-s}{2}+\frac{k}{2}\right) L\left(1-s, \operatorname{sym}^{2} f\right) .
\end{aligned}
$$

We define the following sums:

1) $\Lambda(x):=\sum_{n \leq x} \lambda_{f}(n)$ and its version $A(x):=\sum_{n \leq x} a_{f}(n)$;

2) $C(x):=\sum_{n \leq x} c_{n}$

3) $D(x):=\sum_{n \leq x} d_{n}-R x$.

It has been conjectured that

$$
\Lambda(x) \ll x^{\frac{1}{4}+\varepsilon}, \quad C(x) \ll x^{\frac{1}{3}+\varepsilon}, \quad D(x) \ll x^{\frac{3}{8}+\varepsilon} .
$$

The estimates obtained up until now are essentially less sharp:

$$
\Lambda(x) \ll x^{\frac{1}{3}+\varepsilon} \quad[5], \quad C(x) \ll x^{\frac{1}{2}+\varepsilon} \quad[6], \quad D(x) \ll x^{\frac{3}{5}} \quad[2,3] .
$$

In fact, in the paper [5], dating back to 1933, Walfisz proved a weaker result,

$$
\Lambda(x) \ll x^{\frac{11}{24}+\varepsilon},
$$

but only because Deligne's result was not known in 1933.

It is very important to investigate the mean values of these sums and to estimate the integral

$$
\int_{0}^{x} \Delta_{\ell}^{(f)}(y)^{2} d y \quad(\ell=2,3,4)
$$

Here, for convenience, we use the unifying notation

$$
\Lambda(x)=: \Delta_{2}^{(f)}(x), \quad C(x)=: \Delta_{3}^{(f)}(x), \quad D(x)=: \Delta_{4}^{(f)}(x) .
$$

This emphasizes an unquestionable similarity between our sums and the functions $\Delta_{\ell}(x)$ that are well known in divisor problems (see below). It may be conjectured that, for $\ell=2,3,4$,

$$
\int_{0}^{x} \Delta_{\ell}^{(f)}(y)^{2} d y \sim C_{\ell}(f) x^{(2 \ell-1) / \ell} \quad(x \rightarrow \infty),
$$

where $C_{\ell}(f)>0$. 
For $\ell=2$, Walfisz [5] proved a version of (1.1). He considered arbitrary cuspforms of weight $k \geq 2$ for $\Gamma(N)$. If $f \in S_{k}(\Gamma)^{+}$, from Walfisz's result it follows that

$$
\int_{0}^{x} A(y)^{2} d y=\frac{1}{(4 k+2) \pi^{2}} \sum_{n=1}^{\infty} \frac{a_{f}(n)^{2}}{n^{k+1 / 2}} \cdot x^{k+1 / 2}+O\left(x^{k} \log ^{2} x\right) .
$$

The asymptotics (1.2) was rediscovered in [7 (see Lemma 3 below) and was proved with a slightly worse error term $O\left(x^{k} \log ^{5} x\right)$ in [8, 9. Only recently it was somewhat improved in [10] (with the error term $O\left(x^{k} \log x\right)$ ).

The Voronoi formula for $C(x)$, proved in [6, leads (see [11]) to the estimate

$$
\int_{0}^{x} C(y)^{2} d y \ll x^{\frac{5}{3}+\varepsilon} .
$$

Theorem 1 in [7] gives a better result: $\cdots \ll x^{5 / 3} \log ^{\varkappa} x$ with some constant $\varkappa>0$.

The Voronoi formula for $D(x)$ was obtained in [12]; this formula yields the estimate

$$
\int_{0}^{x} D(y)^{2} d y \ll x^{1+2 \beta+\varepsilon}
$$

with $\beta=\frac{1}{2}$. Recently, Ivić [13] proved that (1.3) is valid with

$$
\beta=\frac{2}{5-2 \mu\left(\frac{1}{2}\right)},
$$

where $\mu(\sigma)$ is the lower bound of all numbers $\xi$ such that

$$
\zeta(\sigma+i t) \ll|t|^{\xi} \text {. }
$$

It is known (Huxley, 2005) that

$$
\mu\left(\frac{1}{2}\right) \leq \frac{32}{205}
$$

but even under the Lindelöf hypothesis $\left(\mu\left(\frac{1}{2}\right)=0\right)$ the result (1.3) gives only the estimate $\cdots \ll x^{(9 / 5)+\varepsilon}$, which is appreciably weaker than the conjectured $\cdots \ll x^{7 / 4}$.

Our goal in the present paper is to obtain the true order of $\int_{0}^{x} C(y)^{2} d y$.

The main result looks like this.

Theorem. For all $x \geq 2$ we have

$$
K_{1} x^{5 / 3} \leq \int_{0}^{x} C(y)^{2} d y \leq K_{2} x^{5 / 3}
$$

where $K_{1}, K_{2}$ are some positive constants.

The proof is presented in $\S \S 2,3$. In $\S 4$ we discuss the problems in question, and in the rest of the present section we give a brief account of the classical divisor problems (see [14, 15. for the details). Great similarity with the above material can be observed.

We recall the formulas

$$
\zeta(s):=\prod_{p}\left(1-p^{-s}\right)^{-1}=\sum_{n=1}^{\infty} n^{-s} \quad(\sigma>1), \quad \zeta(s)^{\ell}=\sum_{n=1}^{\infty} d_{\ell}(n) n^{-s} \quad(\sigma>1),
$$

where $\ell=2,3,4, \ldots$, and $d_{\ell}(n)$ denotes the number of ways of expressing $n$ as a product of $\ell$ factors. The function $\zeta(s)$ admits analytic continuation to the entire complex plane and its only singularity is a simple pole with residue 1 at $s=1$. This function satisfies the functional equation

$$
\pi^{-\frac{1}{2} s} \Gamma\left(\frac{s}{2}\right) \zeta(s)=\pi^{-\frac{1}{2}(1-s)} \Gamma\left(\frac{1-s}{2}\right) \zeta(1-s) .
$$


It is clear that all the $L$-functions considered in this section belong to the Selberg class $S$, and that $\zeta(s)^{2}$ and $L(s, f)$ are of degree two, $\zeta(s)^{3}$ and $L\left(s, \mathrm{sym}^{2} f\right)$ are of degree three, and $\zeta(s)^{4}$ and $L(s, f \times f)$ are of degree four. The definition of the degree in the Selberg class $S$ can be found in [16].

It is known that

$$
\sum_{n \leq x} d_{\ell}(n)=x P_{\ell}(\log x)+\Delta_{\ell}(x),
$$

where $P_{\ell}$ is a polynomial of degree $\ell-1$ and

$$
\Delta_{\ell}(x) \ll x^{1-1 / \ell} \log ^{\ell-2} x \quad(\ell=2,3,4, \ldots) .
$$

This simplest elementary result has been improved many times, especially in the cases where $\ell=2$ (the Dirichlet divisor problem ) and $\ell=3$ (the Piltz divisor problem). We can define the order $\alpha_{\ell}$ of $\Delta_{\ell}(x)$ as the smallest number such that

$$
\Delta_{\ell}(x) \ll x^{\alpha_{\ell}+\varepsilon}
$$

for every $\varepsilon>0$. The exact value of $\alpha_{\ell}$ is not known for any $\ell$; it may be conjectured that

$$
\alpha_{\ell}=\frac{\ell-1}{2 \ell} \text {. }
$$

Also, it may be conjectured that

$$
\int_{0}^{x} \Delta_{\ell}(y)^{2} d y \sim B_{\ell} x^{(2 \ell-1) / \ell} \quad(x \rightarrow \infty),
$$

where $B_{\ell}>0$. So far, this formula has been justified only for $\ell=2$ (Cramér [17) and for $\ell=3$ (Tong [18, III]); see $\S 4$ for the details.

For $\ell=4$, the upper bound

$$
\int_{0}^{x} \Delta_{4}(y)^{2} d y \ll x^{\frac{7}{4}+\varepsilon}
$$

is known (Heath-Brown [19]). For $\ell \geq 5$ we have a collection of estimates

$$
\int_{0}^{x} \Delta_{\ell}(y)^{2} d y \ll x^{\lambda(\ell)}
$$

with $\lambda(\ell)>2-1 / \ell($ see [15, 20]).

An additional remark is in order. Comparison of the corresponding coefficients of $L$-functions of one and the same degree leads to the inequalities $(n=1,2,3, \ldots)$

$$
\left|\lambda_{f}(n)\right| \leq d_{2}(n), \quad\left|c_{n}\right| \leq d_{3}(n), \quad d_{n} \leq d_{4}(n) ;
$$

it is known that $d_{\ell}(n) \ll_{\varepsilon, \ell} n^{\varepsilon}$ for any $\varepsilon>0$.

Notation. We add some notation to that introduced above. $\mathbb{R}$ is the field of real numbers; $\mathbb{C}$ is the field of complex numbers; $K_{3}, K_{4}, K_{5}, \ldots$ are positive constants; $\varepsilon>0$ is an arbitrarily small fixed number.

\section{§2. Proof of the theorem: The lower bound}

In this section we prove the following statement: for every form $f \in S_{k}(\Gamma)^{+}$and all $x \geq 2$ we have

$$
\int_{0}^{x} C(y)^{2} d y \geq K_{1} x^{5 / 3}
$$


In the proof we use (with simplifications) the considerations of the paper [18, I], where a similar problem for the function $\Delta_{\ell}(x), \ell \geq 2$, was studied. Essentially, we deduce and apply an analog of the Voronoi formula for $C(x)$. Below we need the functional equation for $L\left(s, \operatorname{sym}^{2} f\right)$ in the following form. Let $\Phi_{k}(s):=s(s+1) \cdots(s+k)$ $=\Gamma(s+k+1) / \Gamma(s)$,

$$
f(k, s):=\frac{1}{2 \pi i} 2^{2-3 s} \pi^{-3 s} \frac{\Phi_{k-2}(s)}{\Phi_{k-2}(1-s)} \Gamma(s)^{3} \sin \left(\frac{1}{2} \pi s\right) \sin (\pi s) .
$$

Then

$$
\frac{1}{2 \pi i} L\left(1-s, \operatorname{sym}^{2} f\right)=f(k, s) L\left(s, \operatorname{sym}^{2} f\right) .
$$

Let $C_{j}(j=0,1,2, \ldots)$ be the oriented polygonal path with the vertices

$$
-i \infty,-i, j+\frac{3}{2}-i, j+\frac{3}{2}+i, i, i \infty
$$

we put

$$
I_{k, j}(n, x):=\int_{C_{j}} \frac{x^{j+1-s}}{n^{s}} f(k, s) \frac{\Gamma(1-s)}{\Gamma(j+2-s)} d s,
$$

where $x>0, n=1,2,3, \ldots$.

Lemma 1. Suppose $N \geq 0, h \geq 2$ is an integer, and $\min (x, x+h y)>0$. Then

$$
\begin{aligned}
\int_{0}^{y} \ldots \int_{0}^{y} C\left(x+y_{1}+\right. & \left.\cdots+y_{h}\right) d y_{1} \cdots d y_{h} \\
=L\left(0, \operatorname{sym}^{2} f\right) y^{h} & +\sum_{n \leq N} c_{n} \int_{0}^{y} \cdots \int_{0}^{y} I_{k, 0}\left(n, x+y_{1}+\cdots+y_{h}\right) d y_{1} \cdots d y_{h} \\
& +\sum_{l=0}^{h}(-1)^{h-\ell}\left(\begin{array}{c}
h \\
\ell
\end{array}\right) \sum_{n>N} c_{n} I_{k, h}(n, x+\ell y) .
\end{aligned}
$$

Proof. We denote by $C, C^{\prime}, C^{\prime \prime}$ the oriented polygonal paths with the vertices

$$
\begin{array}{ll}
C: & 2-i \infty, 2-i,-h-\frac{1}{2}-i,-h-\frac{1}{2}+i, 2+i, 2+i \infty \\
C^{\prime}: & -\frac{1}{12}-i \infty,-\frac{1}{12}-i,-h-\frac{1}{2}-i,-h-\frac{1}{2}+i,-\frac{1}{12}+i,-\frac{1}{12}+i \infty \\
C^{\prime \prime}: & \frac{13}{12}-i \infty, \frac{13}{12}-i, h+\frac{3}{2}-i, h+\frac{3}{2}+i, \frac{13}{12}+i, \frac{13}{12}+i \infty .
\end{array}
$$

Using the well-known formula

$$
\frac{1}{2 \pi i} \int_{c-i \infty}^{c+i \infty} \frac{u^{-s} \Gamma(s)}{\Gamma(s+q+1)} d s=\left\{\begin{array}{lll}
\frac{(1-u)^{q}}{\Gamma(q+1)} & \text { if } & 0<u \leq 1 \\
0 & \text { if } & u>1
\end{array}\right.
$$


where $q>0$ and $c>0$ (see [15]), we can write

$$
\begin{aligned}
& \int_{0}^{x} C(u) d u=\int_{0}^{x}\left(\sum_{n \leq u} c_{n}\right) d u=\sum_{n \leq x} c_{n}(x-n) \\
&=\sum_{n=1}^{\infty} \frac{c_{n}}{2 \pi i} \int_{2-i \infty}^{2+i \infty} \frac{x^{s+1}}{n^{s}} \frac{1}{s(s+1)} d s=\frac{1}{2 \pi i} \int_{2-i \infty}^{2+i \infty} L\left(s, \operatorname{sym}^{2} f\right) \frac{x^{s+1}}{s(s+1)} d s \\
& \int_{0}^{y} C\left(x+y_{1}\right) d y_{1}=\int_{0}^{x+y} C(u) d u-\int_{0}^{x} C(u) d u \\
&=\frac{1}{2 \pi i} \int_{2-i \infty}^{2+i \infty} L\left(s, \operatorname{sym}^{2} f\right) \frac{(x+y)^{s+1}-x^{s+1}}{s(s+1)} d s \\
&=L\left(0, \operatorname{sym}^{2} f\right) y+\frac{1}{2 \pi i} \int_{C} L\left(s, \operatorname{sym}^{2} f\right) \frac{(x+y)^{s+1}-x^{s+1}}{s(s+1)} d s .
\end{aligned}
$$

Similarly,

$$
\begin{aligned}
\int_{0}^{y} \cdots \int_{0}^{y} C\left(x+y_{1}+\cdots+y_{h}\right) d y_{1} \cdots d y_{h}-L\left(0, \mathrm{sym}^{2} f\right) y^{h} \\
=\frac{1}{2 \pi i} \int_{C} L\left(s, \operatorname{sym}^{2} f\right)\left\{\int _ { 0 } ^ { y } \cdots \int _ { 0 } ^ { y } \left[\left(x+y+y_{2}+\cdots+y_{h}\right)^{s+1}\right.\right. \\
\\
\left.\left.\quad-\left(x+y_{2}+\cdots+y_{h}\right)^{s+1}\right] d y_{2} \cdots d y_{h}\right\} \frac{1}{s(s+1)} d s \\
=\sum_{\ell=0}^{h}(-1)^{h-\ell}\left(\begin{array}{c}
h \\
\ell
\end{array}\right) \frac{1}{2 \pi i} \int_{C} L\left(s, \operatorname{sym}^{2} f\right)(x+\ell y)^{s+h} \frac{\Gamma(s)}{\Gamma(s+h+1)} d s .
\end{aligned}
$$

To evaluate the integral in (2.3), we use the functional equation (2.2) and the estimate

$$
\int_{-\frac{1}{12}+i t}^{2+i t} L\left(s, \operatorname{sym}^{2} f\right) u^{s+h} \frac{\Gamma(s)}{\Gamma(s+h+1)} d s=o(1) .
$$

We have

$$
\begin{aligned}
\frac{1}{2 \pi i} & \int_{C} L\left(s, \operatorname{sym}^{2} f\right) u^{s+h} \frac{\Gamma(s)}{\Gamma(s+h+1)} d s \\
& =\frac{1}{2 \pi i} \int_{C^{\prime}} L\left(s, \operatorname{sym}^{2} f\right) u^{s+h} \frac{\Gamma(s)}{\Gamma(s+h+1)} d s \\
& =\frac{1}{2 \pi i} \int_{C^{\prime \prime}} L\left(1-s, \operatorname{sym}^{2} f\right) u^{h+1-s} \frac{\Gamma(1-s)}{\Gamma(h+2-s)} d s \\
& =\int_{C^{\prime \prime}} L\left(s, \operatorname{sym}^{2} f\right) u^{h+1-s} f(k, s) \frac{\Gamma(1-s)}{\Gamma(h+2-s)} d s \\
& =\sum_{n=1}^{\infty} c_{n} \int_{C^{\prime \prime}} \frac{u^{h+1-s}}{n^{s}} f(k, s) \frac{\Gamma(1-s)}{\Gamma(h+2-s)} d s \\
& =\sum_{n=1}^{\infty} c_{n} \int_{C_{h}} \frac{u^{h+1-s}}{n^{s}} f(k, s) \frac{\Gamma(1-s)}{\Gamma(h+2-s)} d s \\
& =\sum_{n=1}^{\infty} c_{n} I_{k, h}(n, u),
\end{aligned}
$$


where we have also used the estimate

$$
\int_{i t}^{\frac{13}{12}+i t} \frac{u^{h+1-s}}{n^{s}} f(k, s) \frac{\Gamma(1-s)}{\Gamma(h+2-s)} d s=o(1) .
$$

For $j=0,1,2, \ldots$, if $\min (u, u+y)>0$, then

$$
\begin{aligned}
\int_{0}^{y} & I_{k, j}\left(n, u+y_{1}\right) d y_{1} \\
& =\int_{0}^{y}\left[\int_{C_{j}} \frac{\left(u+y_{1}\right)^{j+1-s}}{n^{s}} f(k, s) \frac{\Gamma(1-s)}{\Gamma(j+2-s)} d s\right] d y_{1} \\
& =\int_{0}^{y}\left[\int_{C_{j+1}} \frac{\left(u+y_{1}\right)^{j+1-s}}{n^{s}} f(k, s) \frac{\Gamma(1-s)}{\Gamma(j+2-s)} d s\right] d y_{1} \\
& =\int_{C_{j+1}} \frac{(u+y)^{j+2-s}-u^{j+2-s}}{n^{s}} f(k, s) \frac{\Gamma(1-s)}{\Gamma(j+3-s)} d s \\
& =I_{k, j+1}(n, u+y)-I_{k, j+1}(n, u) .
\end{aligned}
$$

With the help of (2.5), we prove the relation

$$
\begin{gathered}
\int_{0}^{y} \cdots \int_{0}^{y} I_{k, 0}\left(n, x+y_{1}+\cdots+y_{h}\right) d y_{1} \cdots d y_{h} \\
=\sum_{\ell=0}^{h}(-1)^{h-\ell}\left(\begin{array}{l}
h \\
\ell
\end{array}\right) I_{k, h}(n, x+\ell y) .
\end{gathered}
$$

The lemma follows from (2.3), (2.4), and (2.6).

Lemma 2. We have

$$
I_{k, j}(n, x)=b_{j} \frac{x^{\frac{2}{3} j+\frac{1}{3}}}{n^{\frac{1}{3} j+\frac{2}{3}}} \cos \left(6 \pi(n x)^{\frac{1}{3}}-\frac{\pi}{2} j\right)+O\left(\frac{x^{\frac{2}{3} j}}{n^{\frac{1}{3} j+1}}\right) \quad(n=1,2,3, \ldots)
$$

where

$$
b_{j}=\frac{1}{2^{j} \pi^{j+1} 3^{\frac{1}{2}}}
$$

If we take the functional equation for $L\left(s, \mathrm{sym}^{2} f\right)$ in the form (2.2), then the proof of Lemma 2 is completely similar to that of Theorem 2 in [18, I].

We also note that, in a different form, Lemma 2 was formulated in the author's paper [6] as a particular case of a general result by Hafner; see Theorem A, Theorem B, and Lemma 2.1 in Hafner's paper 21 .

Now we pass to the proof of (2.1). Suppose $x \leq X \leq 2 x, 1 \leq u \leq x$, and $h \geq 2$ is an integer. By Lemmas 1 and 2, relation (2.5), and the inequality $L\left(0, \operatorname{sym}^{2} f\right)>0$, we 
have

$$
\begin{aligned}
\int_{0}^{u}\left\{\int_{0}^{y} \ldots \int_{0}^{y} C\left(X+y_{1}+\cdots+y_{h}\right) d y_{1} \cdots d y_{h}\right\} d y \\
=\int_{0}^{u}\left\{L\left(0, \operatorname{sym}^{2} f\right) y^{h}+\sum_{l=0}^{h}(-1)^{h-l}\left(\begin{array}{c}
h \\
\ell
\end{array}\right) \sum_{n=1}^{\infty} c_{n} I_{k, h}(n, X+\ell y)\right\} d y \\
=\frac{1}{h+1} L\left(0, \operatorname{sym}^{2} f\right) u^{h+1}+u\left\{(-1)^{h} I_{k, h}(1, X)+(-1)^{h} \sum_{n=2}^{\infty} c_{n} I_{k, h}(n, X)\right\} \\
\quad+\sum_{\ell=1}^{h} \frac{(-1)^{h-\ell}}{\ell}\left(\begin{array}{c}
h \\
\ell
\end{array}\right) \sum_{n=1}^{\infty} c_{n}\left\{I_{k, h+1}(n, X+\ell u)-I_{k, h+1}(n, X)\right\} \\
\geq u\left\{(-1)^{h} b_{h} X^{\frac{1}{3}+\frac{2}{3} h} \cos \left(6 \pi X^{\frac{1}{3}}-\frac{\pi}{2} h\right)-b_{h} X^{\frac{1}{3}+\frac{2}{3} h} \sum_{n=2}^{\infty} \frac{\left|c_{n}\right|}{\left.n^{\frac{2}{3}+\frac{1}{3} h}+O\left(x^{\frac{2}{3} h}\right)\right\}}\right. \\
\quad-\left\{\sum_{\ell=1}^{h}\left(\begin{array}{l}
h \\
\ell
\end{array}\right) \sum_{n=1}^{\infty} \frac{\left|c_{n}\right|}{\left.n^{\frac{2}{3}+\frac{1}{3}(h+1)} 2 b_{h+1}(X+\ell u)^{\frac{1}{3}+\frac{2}{3}(h+1)}+O\left(x^{\frac{2}{3}(h+1)}\right)\right\}}\right. \\
\geq X^{\frac{1}{3}+\frac{2}{3}(h+1)}\left\{\frac{u}{X^{\frac{2}{3}}} b_{h}\left[1+(-1)^{h} \cos \left(6 \pi X^{\frac{1}{3}}-\frac{\pi}{2} h\right)-\zeta^{3}\left(\frac{2}{3}+\frac{1}{3} h\right)\right]\right. \\
\left.\quad-b_{h+1} 2^{h+1}(h+1)^{h+1} \zeta^{3}\left(1+\frac{1}{3} h\right)+O\left(x^{-\frac{1}{3}-\frac{2}{3}} u\right)+O\left(x^{-\frac{1}{3}}\right)\right\} .
\end{aligned}
$$

For a natural number $m$, let $X_{m}$ denote the root of the equation $6 X^{1 / 3}-h / 2=m$; we have

$$
3\left(X_{m+2}^{\frac{1}{3}}-X_{m}^{\frac{1}{3}}\right)=1, \quad X_{m+2}-X_{m} \sim X_{m}^{\frac{2}{3}} .
$$

For $x \gg 1$ there always exists an $m$ such that

$$
x+1 \leq X_{m}<X_{m+1} \leq x+2 x^{\frac{2}{3}} .
$$

Out of the two numbers $X_{m}$ and $X_{m+1}$, belonging to the interval $\left[x+1, x+2 x^{2 / 3}\right]$, we choose the number (to be denoted by $X_{q}$ ) for which

$$
(-1)^{h} \cos \left(6 \pi X_{q}^{\frac{1}{3}}-\frac{\pi}{2} h\right)=1 \text {. }
$$

We let $h \geq 2$ satisfy the condition

$$
\zeta^{3}\left(\frac{2}{3}+\frac{1}{3} h\right)<2 .
$$

Set $u=c^{\prime} x^{2 / 3}$, where $c^{\prime}$ is a positive constant such that

$$
c^{\prime} b_{h}\left\{2-\zeta^{3}\left(\frac{2}{3}+\frac{1}{3} h\right)\right\}>b_{h+1} 2^{h+1}(h+1)^{h+1} \zeta^{3}\left(1+\frac{1}{3} h\right) .
$$

We have proved the inequality

$$
\int_{0}^{c^{\prime} x^{2 / 3}}\left\{\int_{0}^{y} \cdots \int_{0}^{y} C\left(X_{q}+y_{1}+\cdots+y_{h}\right) d y_{1} \cdots d y_{h}\right\} d y \gg x^{\frac{1}{3}+\frac{2}{3}(h+1)} .
$$

Set $\widetilde{c}=h c^{\prime}+2$. Using (2.7), we obtain

$$
\begin{aligned}
x^{\frac{1}{3}+\frac{2}{3}(h+1)} & \ll \int_{0}^{\tilde{c} x^{2 / 3}}\left|C\left(x+y_{1}\right)\right| d y_{1} \int_{0}^{c^{\prime} x^{2 / 3}}\left\{\int_{0}^{y} d y_{2} \ldots \int_{0}^{y} d y_{h}\right\} d y \\
& \ll x^{\frac{2}{3} h} \int_{x}^{x+\widetilde{c} x^{2 / 3}}|C(y)| d y,
\end{aligned}
$$


which implies the inequality

$$
x \ll \int_{x}^{x+\widetilde{c} x^{2 / 3}}|C(y)| d y .
$$

Next, we have

$$
\int_{0}^{x}|C(y)| d y \geq \sum_{j=0}^{\left[\frac{1}{2} \widetilde{c}^{-1} x^{1 / 3}\right]} \int_{\frac{x}{2}+j \tilde{c} x^{2 / 3}}^{\frac{x}{2}+(j+1) \widetilde{c} x^{2 / 3}}|C(y)| d y \gg x^{\frac{4}{3}} .
$$

We have proven that

$$
\int_{0}^{x}|C(y)| d y \geq K_{3} x^{\frac{4}{3}}
$$

if $x>x_{1}$ and $x_{1}>0$ is sufficiently large. Since $c_{1}=1$, for $2 \leq x \leq x_{1}$ we have

$$
\int_{0}^{x}|C(y)| d y \geq \int_{0}^{2}|C(y)| d y=1 .
$$

It follows that

$$
\int_{0}^{x}|C(y)| d y \geq K_{4} x^{\frac{4}{3}}
$$

for $x \geq 2$. Using (2.8) and the Cauchy inequality, we arrive at (2.1).

\section{§3. Proof of the theorem: The upper bound}

In this section we prove the following statement: for every form $f \in S_{k}(\Gamma)^{+}$, as $x \rightarrow \infty$ we have

$$
\int_{0}^{x} C(y)^{2} d y \ll x^{5 / 3} .
$$

For the proof, apart from the results on the mean value of the error term for the summatory function of the coefficients of a general Dirichlet series (see [7, 10]), we also need some analytic properties of $L$-functions associated with automorphic forms on GL(3) [22]-24].

Let $\{a(n)\}$ and $\{b(n)\}$ be two sequences of complex numbers, not all zero, and let $\left\{\lambda_{n}\right\}$ and $\left\{\mu_{n}\right\}$ be two sequences of real numbers such that

$$
\begin{array}{ll}
0<\lambda_{1}<\lambda_{2}<\lambda_{3}<\cdots & \left(\lambda_{n} \rightarrow \infty\right), \\
0<\mu_{1}<\mu_{2}<\mu_{3}<\cdots & \left(\mu_{n} \rightarrow \infty\right) .
\end{array}
$$

Let $r \in \mathbb{R}$. We put

$$
\Delta(s):=\prod_{\nu=1}^{N} \Gamma\left(\alpha_{\nu} s+\beta_{\nu}\right),
$$

where $N \geq 1, \beta_{\nu} \in \mathbb{C}$, and $\alpha_{\nu}>0$. Let $A:=\sum_{\nu=1}^{N} \alpha_{\nu}$. We assume that the Dirichlet series

$$
\varphi(s):=\sum_{n=1}^{\infty} a(n) \lambda_{n}^{-s}, \quad \psi(s):=\sum_{n=1}^{\infty} b(n) \mu_{n}^{-s}
$$

converge in some half-planes of the $s$-plane, have finite abscissas of absolute convergence, admit analytic continuation to the entire $s$-plane, and satisfy the functional equation

$$
\Delta(s) \varphi(s)=\Delta(r-s) \psi(r-s) .
$$

Also, it is assumed that the set of singularities of the function $\Delta(s) \varphi(s)$ is contained in a compact set $S \subset \mathbb{C}$. For the further assumptions, which are fulfilled practically always in applications, see [7, 10. 
We introduce the notation

$$
Q(x):=\frac{1}{2 \pi i} \int_{C} \frac{\varphi(s)}{s} x^{s} d s,
$$

where $C$ is a curve enclosing all the singularities of the integrand, and the notation

$$
E(x):=\sum_{\lambda_{n} \leq x}^{\prime} a(n)-Q(x),
$$

where the dash indicates that the last term must be multiplied by $1 / 2$ if $\lambda_{n}=x$. $E(x)$ is called the error term for the summatory function of the coefficients of the Dirichlet series $\varphi(s)$. Upper estimates for the mean value of $E(x)$ are given in the following lemma, part 1) of which was proved in [7] and part 2) in [10].

Lemma 3. 1) If the functional equation (3.2) is satisfied with $r>0, A \geq 1$ and $\mu_{n}=\varkappa^{\prime} n$, $\lambda_{n}=\varkappa^{\prime \prime} n\left(\varkappa^{\prime}, \varkappa^{\prime \prime}\right.$ are positive constants $)$, the only singularities of the function $\varphi$ are poles, and

$$
\sum_{\mu_{n} \leq x}|b(n)|^{2} \ll x^{2 a-1} \log ^{b} x
$$

then for $2 a-r-1 / A \leq 0$ we have

$$
\int_{0}^{x}|E(y)|^{2} d y=K_{5} x^{2 d+1}+O\left(x^{2 d+1 /(2 A)} \log ^{b+2} x\right) \quad(x \rightarrow \infty),
$$

where $d=r / 2-1 /(4 A)$.

If $2 a-r-1 / A>0$, then, assuming additionally that

$$
\sum_{\lambda_{n} \leq x}|a(n)|^{2} \ll x^{2 a-1} \log ^{b} x
$$

as $x \rightarrow \infty$ and that $b \geq 2(\rho-1)$, where $\rho$ is the maximal order of the pole with the maximal real part, we have

$$
\int_{0}^{x}|E(y)|^{2} d y \ll x^{2 d+1}+x^{2 a+1-2 / A} \log ^{b+1} x \quad(x \rightarrow \infty) .
$$

2) Suppose that the assumptions of part 1) are fulfilled; in particular, the above inequalities for the sums

$$
\sum_{\lambda_{n} \leq x}|a(n)|^{2}, \quad \sum_{\mu_{n} \leq x}|b(n)|^{2},
$$

are valid. Moreover, assume that

either $r+3 /(2 A)<2 a<r+5 /(2 A)$,

or $r+3 /(2 A)=2 a$ with $A>1$.

Then

$$
\int_{0}^{x}|E(y)|^{2} d y \ll x^{2 a+1-2 / A} \log ^{b} x \quad(x \rightarrow \infty) .
$$

Note that below we shall need only part 2) of Lemma 3; part 1) is cited for completeness. We pass to the proof of (3.1). First, we consider the $L$-function $L\left(s, \operatorname{sym}^{2} f\right)$. With any Hecke eigencuspform $f \in S_{k}(\Gamma)^{+}$, we associate the form $F$ on GL(3) with the Fourier coefficients $a(m, n)$ and the $L$-function

$$
L(s, F)=\sum_{n=1}^{\infty} a(1, n) n^{-s}
$$

such that the Euler $p$-factors of the $L$-functions $L(s, F)$ and $L\left(s, \operatorname{sym}^{2} f\right)$ coincide. In other words, we have

$$
L(s, F)=L\left(s, \operatorname{sym}^{2} f\right) .
$$


The form $F$ is called the Gelbart-Jacquet lift (adjoint square lift); the existence of it was established in 22. It is known that the function $L(s, F)$ is entire and $L(1, F)>0$.

Let $L(s, F \times F)$ denote the $L$-function of the Rankin-Selberg convolution of the form $F$ with itself.

We recall (see $\S 1)$ that

$$
L(s, f)=\prod_{p}\left(1-\alpha p^{-s}\right)^{-1}\left(1-\bar{\alpha} p^{-s}\right)^{-1}
$$

(for simplicity we set $\alpha_{p}=: \alpha$ ); then $L(s, F \times F$ ) has the Euler product

$$
\begin{aligned}
L(s, F \times F)=\prod_{p} & \left(1-\alpha^{4} p^{-s}\right)^{-1}\left(1-\alpha^{2} p^{-s}\right)^{-1}\left(1-p^{-s}\right)^{-1} \\
& \times\left(1-\alpha^{2} p^{-s}\right)^{-1}\left(1-p^{-s}\right)^{-1}\left(1-\bar{\alpha}^{2} p^{-s}\right)^{-1}\left(1-p^{-s}\right)^{-1} \\
& \times\left(1-\bar{\alpha}^{2} p^{-s}\right)^{-1}\left(1-\bar{\alpha}^{4} p^{-s}\right)^{-1} .
\end{aligned}
$$

Analytic continuability and the functional equation for $L(s, F \times F)$, relating $s$ and $1-s$, were studied by several authors; see [22]-24].

The symmetric square $L$-function of $F$ is given by the Euler product

$$
\begin{aligned}
L\left(s, \operatorname{sym}^{2} F\right):=\prod_{p} & \left(1-\alpha^{4} p^{-s}\right)^{-1}\left(1-\alpha^{2} p^{-s}\right)^{-1}\left(1-p^{-s}\right)^{-1} \\
& \times\left(1-p^{-s}\right)^{-1}\left(1-\bar{\alpha}^{2} p^{-s}\right)^{-1}\left(1-\bar{\alpha}^{4} p^{-s}\right)^{-1} .
\end{aligned}
$$

We have

$$
L(s, F \times F)=L(s, F) L\left(s, \operatorname{sym}^{2} F\right) .
$$

In a very general situation, Bump and Ginzburg [24] showed that if $f$ is not a lift in GL(1) (this is true if $\left.f \in S_{k}(\Gamma)^{+}\right)$, then $L\left(s, \operatorname{sym}^{2} F\right.$ ) has a simple pole at $s=1$ and is analytic elsewhere. Relation (3.4) and the properties of $L(s, F)$ show that $L(s, F \times F)$ also has a simple pole at $s=1$ and is analytic elsewhere.

Now we deduce the asymptotics

$$
\sum_{n \leq x} c_{n}^{2}=K_{6} x+O\left(x^{\gamma}\right) \quad(x \rightarrow \infty),
$$

where $\gamma<1$ is a constant.

For the proof, we expand the function

$$
T(s):=\sum_{n=1}^{\infty} c_{n}^{2} n^{-s}
$$

in the Euler product

$$
T(s)=\prod_{p}\left\{1+\left(\alpha^{2}+1+\bar{\alpha}^{2}\right)^{2} p^{-s}+\left(\alpha^{4}+\alpha^{2}+2+\bar{\alpha}^{2}+\bar{\alpha}^{4}\right)^{2} p^{-2 s}+\cdots\right\} .
$$

Comparing this with the Euler product of the $L$-function $L(s, F \times F)$, we see that

$$
T(s)=L(s, F \times F) \cdot B(s) \quad(\sigma>1),
$$

where

$$
B(s)=\prod_{p}\left(1+\frac{A_{2}}{p^{2 s}}+\frac{A_{3}}{p^{3 s}}+\cdots\right) .
$$

Let $P_{0}:=\prod_{p \leq p_{0}} p$; if in every Euler product of relation (3.6) we restrict ourselves to the $p$-factors with $\left(p, P_{0}\right)=1$, we obtain an analog of $(3.6)$ :

$$
T^{(0)}(s)=L^{(0)}(s, F \times F) \cdot B^{(0)}(s) .
$$


It can be shown that for any $\varepsilon>0$ there exists a prime $p_{0}=p_{0}(\varepsilon)$ such that, for $\sigma \geq(1 / 2)+\varepsilon$,

$$
1 \gg\left|B^{(0)}(s)\right| \gg 1 \text {. }
$$

Therefore, by $(3.7), T^{(0)}(s)$ admits analytic continuation to the half-plane $\sigma \geq(1 / 2)+\varepsilon$ with one singularity, a simple pole at $s=1$. Therefore, $T(s)$ admits analytic continuation to the half-plane $\sigma>1 / 2$ with one singularity, a simple pole at $s=1$ (the latter fact agrees with the asymptotics (3.8) below). From the properties of $L(s, F \times F)$, it follows that the growth of this function in the critical strip is at most polynomial in $|t|$. Therefore, $T(s)$ possesses a similar growth in the half-plane $\sigma \geq(1 / 2)+\varepsilon$.

Now the asymptotics (3.5) can be obtained with the help of the Perron inversion formula. The function $T(s)$ has in fact a simple pole at $s=1$, because, by the Ikehara theorem, we have

$$
\sum_{\substack{n \leq x \\\left(n, P_{0}\right)=1}} c_{n}^{2} \sim K_{7} x \quad(x \rightarrow \infty) .
$$

In our case formula (3.3) reduces to

$$
E(x)=\sum_{n \leq x}^{\prime} c_{n}-L\left(0, \operatorname{sym}^{2} f\right) .
$$

Taking $r=1, A=3 / 2, a=1$, and $b=0$, and using part 2) of Lemma 3 , we see that

$$
\int_{0}^{x}|E(y)|^{2} d y \ll x^{5 / 3} \quad(x \rightarrow \infty) .
$$

Statement (3.1) follows; combining this with (2.1), we complete the proof of the theorem.

\section{$\S 4$. REMARKS}

In this section we discuss some problems, in particular, the possibility of obtaining the asymptotics in the main result of our paper. In its turn, this problem is related to the open question as to whether $x^{-1 / 3} C(x)$ has a distribution function (see [11). Taking a certain similarity between $C(x)$ and $\Delta_{3}(x)$ into account, we formulate Tong's important result [18, III]. Tong proved the relation

$$
\int_{0}^{x} \Delta_{\ell}(y)^{2} d y=\left((4 \ell-2) \pi^{2}\right)^{-1} \sum_{n=1}^{\infty} d_{\ell}(n)^{2} n^{-(\ell+1) / \ell} \cdot x^{(2 \ell-1) / \ell}+R_{\ell}(x),
$$

where

$$
R_{\ell}(x) \ll \begin{cases}x \log ^{5} x & (\ell=2), \\ x^{\gamma_{\ell}+\varepsilon}, \gamma_{\ell}=2-\frac{3-4 \sigma_{\ell}}{2 \ell\left(1-\sigma_{\ell}\right)-1} & (\ell \geq 3),\end{cases}
$$

and $\sigma_{\ell}$ is the lower bound of all $\sigma$ such that

$$
\int_{1}^{T}|\zeta(\sigma+i t)|^{2 \ell} d t \ll T^{1+\varepsilon}
$$

for every $\varepsilon>0$; here the condition $\sigma_{\ell} \leq(\ell+1) /(2 \ell)$ is imposed.

For $\ell=2$, Tong refined Cramér's initial result [17, 15] substantially. Consider the case where $\ell=3$. The first nontrivial result $\sigma_{3} \leq 2 / 3$ (a particular case of Titchmarsh's general result $\sigma_{\ell} \leq(\ell-1) / \ell$, see [25]) is not sufficient for obtaining the asymptotics with the help of (4.1), but the result $\sigma_{3} \leq 5 / 8$ by Davenport [26] suffices. By proving the inequality $\sigma_{3} \leq 7 / 12$, Ivić [27] improved Tong-Davenport's asymptotics. 
Note that (4.1) and the Lindelöf hypothesis lead to the asymptotics at once for all $\ell \geq 2$, because the Lindelöf hypothesis is equivalent to the statement that

$$
\int_{1}^{T}\left|\zeta\left(\frac{1}{2}+i t\right)\right|^{2 \ell} d t \ll T^{1+\varepsilon} \quad(\ell=1,2,3, \ldots)
$$

(see [14]).

It may be conjectured that an analog of (4.1) (for $\ell=3$ ) is valid for $C(x)$, but this has not been proved as yet. Also, the quantity $\sigma\left(\operatorname{sym}^{2} f\right)$ needs an estimation; this is the lower bound of all $\sigma$ such that

$$
\int_{1}^{T}\left|L\left(\sigma+i t, \operatorname{sym}^{2} f\right)\right|^{2} d t \ll T^{1+\varepsilon}
$$

for every $\varepsilon>0$. After Ivić's paper [27, it became clear that the mean value theorem for Dirichlet polynomials [15, Theorem 5.2] makes it possible to unify the approach to similar problems for $L$-functions belonging to the Selberg class $S$. The best estimate obtained in this way is (see [28])

$$
\sigma\left(\operatorname{sym}^{2} f\right) \leq \frac{2}{3}
$$

In conclusion, we formulate several conjectures. Let $\theta_{\ell}(\ell=2,3,4, \ldots)$ be the smallest number such that

$$
R_{\ell}(x) \ll x^{\theta_{\ell}+\varepsilon} .
$$

Analyzing the proof of Theorem 5 in [18, I], we may conjecture that

$$
\theta_{\ell}=2\left(1-\frac{1}{\ell}\right)
$$

Similarly, analyzing the proof of inequality (2.1), we may conjecture that

$$
\int_{0}^{x} C(y)^{2} d y=C_{3}(f) x^{\frac{5}{3}}+O\left(x^{\frac{4}{3}+\varepsilon}\right) \quad(x \rightarrow \infty),
$$

where the error term cannot be reduced to $O\left(x^{\gamma}\right), \gamma<4 / 3$.

In a similar way, we arrive at the following conjecture: the error term in the asymptotics

$$
\int_{0}^{x} \Lambda(y)^{2} d y=C_{2}(f) x^{\frac{3}{2}}+O(x \log x)
$$

(see [10]) cannot be reduced to $O\left(x^{\gamma_{1}}\right), \gamma_{1}<1$.

Note two moments: 1) in the book [15, the following conjecture was stated: $\theta_{\ell}=$ $(3 / 2)(1-1 / \ell) ; 2)$ in $[29$, the estimate

$$
R_{2}(x)=\Omega_{-}\left(x \log ^{2} x\right)
$$

was proved.

\section{REFERENCES}

[1] G. Shimura, On the holomorphy of certain Dirichlet series, Proc. London Math. Soc. (3) 31 (1975), 79-98. MR0382176 (52:3064)

[2] R. A. Rankin, Contributions to the theory of Ramanujan's function $\tau(n)$ and similar arithmetical functions. II. The order of the Fourier coefficients of the integral modular forms, Proc. Cambridge Philos. Soc. 35 (1939), 357-372. MR0000411(1:69d)

[3] A. Selberg, Bemerkungen über eine Dirichletsche Reihe, die mit der Theorie der Modulformen nahe verbunden ist, Arch. Math. Naturvid. 43 (1940), 47-50. MR0002626 (2:88a)

[4] S. Gelbart and F. Shahidi, Analytic properties of automorphic L-functions, Acad. Press, Inc., Boston, MA, 1988. MR0951897 (89f:11077)

[5] A. Walfisz, Über die Koeffizientensummen einiger Modulformen, Math. Ann. 108 (1933), 75-90. MR 1512835 
[6] O. M. Fomenko, Identities involving the coefficients of automorphic L-functions, Zap. Nauchn. Sem. S.-Peterburg. Otdel. Mat. Inst. Steklov. (POMI) 314 (2004), 247-256; English transl., J. Math. Sci. (N.Y.) 133 (2006), no. 6, 1749-1755. MR2119744 (2005m:11093)

[7] K. Chandrasekharan and R. Narasimhan, On the mean value of the error term of a class of arithmetical functions, Acta Math. 112 (1964), 41-67. MR0160765 (28:3976)

[8] M. Jutila, Lectures on a method in the theory of exponential sums, Tata Inst. Fund. Res. Lectures on Math. and Phys., vol. 80, Springer-Verlag, Berlin, 1987. MR0910497 (89g:11069)

[9] A. Ivić, Large values of certain number-theoretic error terms, Acta Arith. 56 (1990), 135-159. MR:1075641 (91j:11078)

[10] Y.-K. Lau, On the mean square formula of the error term for a class of arithmetical functions, Monatsh. Math. 128 (1999), 111-129. MR.1712484 (2000h:11107)

[11] O. M. Fomenko, The behavior of Riesz means of the coefficients of a symmetric square L-function, Zap. Nauchn. Sem. S.-Peterburg. Otdel. Mat. Inst. Steklov. (POMI) 337 (2006), 274-286; English transl., J. Math. Sci. (N.Y.) 143 (2007), no. 3, 3174-3181. MR.2271968 (2007h:11059)

[12] A. Ivić, K. Matsumoto, and Y. Tanigawa, On Riesz means of the coefficients of the Rankin-Selberg series, Math. Proc. Cambridge Philos. Soc. 127 (1999), 117-131. MR.1692491 (2000c:11068)

[13] A. Ivić, On some mean square estimates in the Rankin-Selberg problem, Appl. Anal. Discrete Math. 1 (2007), 111-121. MR2316591

[14] E. C. Titchmarsh, The theory of the Riemann zeta-function, 2nd ed., Clarendon Press, Oxford Univ. Press, New York, 1986. MR0882550 (88c:11049)

[15] A. Ivić, The Riemann zeta-function, Wiley, New York, 1985. MR0792089 (87d:11062)

[16] A. Selberg, Old and new conjectures and results about a class of Dirichlet series, Proceedings of the Amalfi Conference on Analytic Number Theory (Maiori, 1989), Univ. Salerno, Salerno, 1992, pp. 367-385; Collected papers. Vol. 2, Springer-Verlag, Berlin, 1991, pp. 47-63. MR1220477 (94f:11085) MR:1295844 (95g:01032)

[17] H. Cramér, Über zwei Sätze des Herrn G. H. Hardy, Math. Z. 15 (1922), 201-210. MR.1544568

[18] K.-C. Tong, On divisor problems. I, III, Acta Math. Sinica 5 (1955), 313-324; 6 (1956), 515-541. (Chinese) MR0073632(17:462c) MR0098718(20:5173)

[19] D. R. Heath-Brown, Mean values of the zeta function and divisor problems, Recent Progress in Analytic Number Theory, Vol. 1 (Durham, 1979), Acad. Press, London-New York, 1981, pp. 115119. MR0637345 (83c:10057)

[20] A. de Roton, On the mean square of the error term for an extended Selberg class, Acta Arith. 126 (2007), 27-55. MR.2284311 (2007j:11121)

[21] J. L. Hafner, On the representation of the summatory functions of a class of arithmetical functions, Analytic Number Theory (Philadelphia, 1980), Lecture Notes in Math., vol. 899, Springer, BerlinNew York, 1981, pp. 148-165. MR0654524 (83g:10030)

[22] S. Gelbart and H. Jacquet, A relation between automorphic representations of GL(2) and GL(3), Ann. Sci. École Norm. Sup. (4) 11 (1978), 471-542. MR0533066 (81e:10025)

[23] J. Hoffstein and P. Lockhart, Coefficients of Maass forms and the Siegel zero, Ann. of Math. (2) 140 (1994), 161-181. MR 1289494 (95m:11048)

[24] D. Bump and D. Ginzburg, Symmetric square L-functions on GL(r), Ann. of Math. (2) 136 (1992), 137-205. MR 1173928 (93i:11058)

[25] E. C. Titchmarsh, The zeta-function of Riemann, Cambridge Univ. Press, London, 1930.

[26] H. Davenport, Note on mean-value theorems for the Riemann zeta-function, J. London Math. Soc. 10 (1935), 136-138.

[27] A. Ivić, On mean values of some zeta-functions in the critical strip, J. Théor. Nombres Bordeaux 15 (2003), 163-178. MR2019009 (2004i:11097)

[28] K. Matsumoto, Liftings and mean value theorems for automorphic L-functions, Proc. London Math. Soc. (3) 90 (2005), 297-320. MR2142129 (2006f:11053)

[29] Y.-K. Lau and K.-M. Tsang, Mean square of the remainder term in the Dirichlet divisor problem, J. Théor. Nombres Bordeaux 7 (1995), 75-92. MR1413567 (98k:11126)

St. Petersburg Branch, Steklov Mathematical Institute, Russian Academy of Sciences, Fontanka 27, St. Petersburg 191023, Russia

E-mail address: fomenko@pdmi.ras.ru

Received 5/APR/2007

Translated by THE AUTHOR 